[ Review article ]

\title{
Summary 2013 ESC guidelines
}

Marc J. CLAEYS ${ }^{1}$ MD, PhD; Philippe VAN DE BORNE², MD, PhD; Georges H. MAIRESSE ${ }^{3}$, MD; Guy VAN

CAMP, MD, PhD

${ }^{1}$ Antwerp University Hospital, ${ }^{2}$ ULB Brussels, ${ }^{3}$ Cliniques du Sud Luxembourg, Arlon, ${ }^{4}$ UZ Brussels, Belgium.

Keywords ESC-guidelines.

\section{INTRODUCTION}

During the ESC congress in September 2013 in Amsterdam, the new ESC guidelines were presented and are now available on the ESC website (http://www.escardio.org/guidelines).

The new guidelines cover management recommendations on the following cardiovascular items: stable coronary artery disease, hypertension and cardiac pacing.

The present document gives a summary of these guidelines and highlights the most important recommendations and changes in the management of these diseases. It will help to increase awareness about the new guidelines and may stimulate to consult the full document for specific items. Ultimately, the authors hope that this document will enhance implementation of the new ESC guidelines in daily clinical practice.

\section{GUIDELINES ON THE MANAGEMENT OF STABLE CORONARY ARTERY DISEASE}

\section{Summary by M. Claeys, MD, PhD, FESC}

This new document, replacing the 2006 guidelines, covers different aspects such as diagnosis, risk stratification and treatment. The most important messages can be summarized as follows:

\section{Address for correspondence:}

Prof. M. Claeys, MD, Dept. of Cardiology, Antwerp University Hospital, Wilrijkstraat 10, B-2650 Edegem, Belgium. E-mail: marc.claeys@ua.ac.be

Received 24 October 2013; accepted for publication 12 November 2013.

\section{Diagnostic evaluation}

The decision to perform a non-invasive diagnostic stress test in patients with chest discomfort is highly dependent on the pre-test probability (PTP). The document provides an algorithm to assess PTP based upon age, sex and clinical presentation (typical angina, less typical angina, non-anginal pain).

For patients with a PTP of more than $85 \%$ (e.g. men $>70 \mathrm{y}$ with typical pain) or less than $15 \%$ (e.g. women $<40 \mathrm{y}$ without typical angina) the diagnosis of the presence or absence of obstructive coronary artery disease (CAD) is sufficiently high and does not warrant additional testing. For patients with an intermediate PTP (15-85\%) additional non-invasive testing is needed. Preferentially, stress imaging testing (echo, SPECT) should be selected if local expertise and availability permits given the superior diagnostic performance of such tests. The standard exercise ECG can still be performed as an initial test for patients with PTP between 15 en $65 \%$ and a good left ventricular function. In young patients the radiation issue should be considered in the selection of the diagnostic stress test. Coronary CT angiography is potentially useful to exclude CAD particularly in patients at low intermediate PTP.

Once the diagnosis of CAD is made, risk stratification is needed to identify high-risk groups that will benefit of revascularization. High-risk groups include patients with estimated CV mortality $>3 \%$ year as evidenced by low ischaemic threshold (on exercise ECG), great area of ischaemia $(>10 \%)$ on stress imaging test, diminished left ventricular function $(<50 \%)$ or extensive proximally located coronary artery disease on coronary CT angiography. Those patients should undergo invasive coronary angiography with revascularization of the suitable lesions. 
For the other patients invasive coronary angiography should be considered in case of inconclusive non-invasive stress tests or in case of inadequate control of symptoms under optimal medical therapy.

\section{Management}

The management of CAD patients aims to reduce symptoms and improve prognosis. Symptom relief can be achieved by starting treatment with a beta blocker or heart rate-lowering calcium channel blocker. In case of insufficient control, combination therapy with long-acting nitrates or with ivabradine can be considered. Event prevention can be achieved by lifestyle modifications, control of CAD risk factors and pharmacologic treatment with aspirin, statins (target LDL chol $<70 \mathrm{mg} \%$ ) and ACE inhibition (for patients with hypertension, cardiac failure, kidney disease or diabetes).

The indications and modalities of revascularization in patients with stable CAD follow the recent recommendations on myocardial revascularization (2010). Beyond relief of symptoms, revascularization will improve also the prognosis in high-risk groups (left main disease, multivessel disease with impaired left ventricular function, large area of ischaemia). PCI is the preferred revascularization approach for patients with single- or double-vessel disease without proximal LAD involvement, whereas CABG is the preferred treatment for complex triple-vessel disease. A heart team approach to revascularization is recommended in patients with unprotected left main artery, multivessel disease, diabetes or major comorbidities.

After coronary stent implantation, dual antiplatelet therapy should be taken for at least 1 month after bare metal stent and 6 to 12 months after $2^{\text {nd }}$ generation drug-eluting stents.

\section{REFERENCE}

1. Task Force Members, Montalescot G, Sechtem U, et al. 2013 ESC guidelines on the management of stable coronary artery disease: The Task Force on the management of stable coronary artery disease of the European Society of Cardiology. Eur Heart J 2013; 34: 2949-3003.

\section{GUIDELINES ON CARDIAC PACING AND CARDIAC RESYNCHRONIZATION THERAPY}

\section{Summary by G. Mairesse, MD, FESC}

These new ESC guidelines have globally simplified the pacing indications, but have also extended CRT indications. Only one third are class I indications and only $10 \%$ have a level of evidence A. For that reason, a rating of the strength of recommendations was used.
These guidelines, however, represent a comprehensive summary to help physicians for decision making in daily practice.

\section{Pacing for bradycardia}

The classification of bradyarrhythmias has now to be based on the patient's clinical presentation: persistent or intermittent bradycardia.

In persistent bradycardia, class I indication for pacing in sinus node disease is accepted when symptoms can clearly be attributed to bradycardia. In acquired AV block, class I indication is achieved in patients with third- or second-degree type $2 \mathrm{AV}$ block, irrespective of symptoms.

In intermittent bradycardia, every effort should be made to document this bradycardia by ECG before considering pacemaker implantation. Pacing is indicated in patients affected by sinus node disease who have the documentation of symptomatic bradycardia due to sinus arrest or sinus-atrial block, in patients with intermittent/ paroxysmal intrinsic third-or second-degree AV block, in patients with $\mathrm{HV}$ interval $>70 \mathrm{msec}$ at electrophysiological study, or in patients with alternating BBB.

In addition, pacing should be considered in patients $>40$ years with recurrent unpredictable reflex syncope and documented symptomatic pauses due to sinus arrest or AV block or both, in patients with a history of syncope and documented asymptomatic pauses $>6 \mathrm{sec}$ of either origin, or in patients with dominant cardioinhibitory carotid sinus syndrome and recurrent unpredictable syncope. It may also be indicated in patients with tiltinduced cardioinhibitory response with recurrent frequent syncope $>40$ years after alternative therapy has failed or in patients with unexplained syncope and positive ATP testing.

With the exception of permanent atrial fibrillation (AF), a dual-chamber pacemaker remains the first choice in all indications. Prevention and termination of atrial tachyarrhythmias does not represent a stand-alone indication for pacing.

During follow-up, magnetic resonance imaging at 1.5 Tesla can be performed with a low risk of complications if manufacturer's instructions are followed or if appropriate precautions are taken. Device-base remote monitoring should be considered in order to provide earlier detection of clinical problems.

\section{Pacing for cardiac resynchronization therapy (CRT)}

The magnitude of the benefit from CRT is clearly higher when QRS are wider, in the presence of LBBB, in females and in non-ischaemic cardiomyopathy. A class 
I indication for CRT is obtained in the presence of LBBB with a QRS duration of $>150 \mathrm{msec}$, in chronic HF patients with left ventricular ejection fraction (LVEF) $<35 \%$ who remain in functional class II or more despite adequate medical therapy. The same indication seems also to apply to patients with a QRS duration of 120-150 msec. It is less clear in non-LBBB patients especially when QRS duration remains 120-150 msec.

In patients with permanent $\mathrm{AF}$, CRT should be considered in chronic HF patients, intrinsic QRS $>120 \mathrm{msec}$ and LVEF $<35 \%$ who remain in functional class III or ambulatory IV despite adequate medical therapy provided a biventricular pacing as close to $100 \%$ as can be achieved, eventually requiring AV junction ablation. In AF patients with uncontrolled heart rate who are candidates for AV junction ablation, CRT should be considered in patients with reduced LVEF. There are no data regarding AF patients in functional class II.

Upgrade from a conventional pacemaker or defibrillator to CRT is also indicated in heart failure patients with LVEF $<35 \%$ and a high percentage of ventricular pacing who remain in functional class II or more despite adequate medical treatment, and should be considered de novo in heart failure patients, reduced LVEF $(<50 \%)$ and expected high percentage of ventricular pacing in order to decrease the risk of worsening heart failure.

The selection of CFRT-P versus CRT-D (back-up ICD) should be based upon the overall clinical condition, taking into account a small survival benefit with CRT-D but at the cost of a higher complication rate.

The goal in CRT should be to achieve biventricular pacing as close to $100 \%$ as possible since the survival benefit and reduction in hospitalization are strongly associated with an increasing percentage of biventricular pacing. Apical position of the LV lead should be avoided when possible and LV lead placement may be targeted at the latest activated LV segment.

\section{REFERENCE}

1. Brignole M, Auricchio A, Baron-Esquivias, et al. 2013 ESC guidelines on cardiac pacing and cardiac resynchronization therapy: the task force on cardiac pacing and resynchronization therapy of the European Society of Cardiology (ESC). Developed in collaboration with the European Heart Rhythm Association (EHRA). Eur Heart J 2013; 15: 1070-118.

\section{GUIDELINES FOR THE MANAGEMENT OF ARTERIAL HYPERTENSION}

\section{Summary by $\mathrm{P}$. van de Borne, MD, PhD, FESC}

This document is the result of the work of the Joint Task Force of the European Society of Cardiology and the European Society of Hypertension. The most important messages can be summarized as follows:

\section{Risk stratification}

The 2013 ESH/ESC Guidelines for the management of arterial hypertension ${ }^{1}$ have reinforced the importance of assessing total cardiovascular (CV) risk in all asymptomatic patients, using the SCORE model. The observation that any of four markers of organ damage (microalbuminuria, arterial stiffness, left ventricular (LV) hypertrophy and carotid plaques) can predict CV mortality independently of SCORE stratification, is a relevant argument in favour of using assessment of organ damage (OD) in daily clinical practice, although more data from larger studies in different populations would be desirable.

The prognostic value of home blood pressure monitoring and of its role for diagnosis and management of hypertension, next to ambulatory blood pressure monitoring, has been strengthened. In masked hypertension (i.e. a condition where BP in the office is normal, but elevated out of the doctor's office), both lifestyle measures and antihypertensive drug treatment should be considered. This type of hypertension has been consistently found to have a CV risk very close to that of in and out of office hypertension.

\section{Organ damage}

Calculation of LV mass (LVM) is currently performed according to the American Society of Echocardiography formula with left ventricular hypertrophy thresholds of $95 \mathrm{~g} / \mathrm{m}^{2}$ for women and $115 \mathrm{~g} / \mathrm{m}^{2}$ (BSA) for men.

The relationship between carotid IMT and CV events is a continuous one and determining a threshold for high $\mathrm{CV}$ risk is rather arbitrary. Presence of a plaque and increased carotid IMT add little to each other for predicting CV events.

In both diabetic and non-diabetic hypertensive patients, micro albuminuria, even below the threshold values usually considered, has been shown to predict $\mathrm{CV}$ events, and continuous relationships between CV, as well as non-CV mortality. An arbitrary threshold for the definition of micro albuminuria has been established as $30 \mathrm{mg} / \mathrm{g}$ of creatinine.

Grade III (retinal haemorrhages, micro aneurysms, hard exudates, cotton wool spots) and grade IV retinopathy (grade III signs and papilloedema and/or macular oedema) are indicative of severe hypertensive retinopathy, with a high predictive value for mortality. Criticism with respect to the reproducibility of grade I and grade II retinopathy has been raised. Fundoscopy is only recommended in resistant hypertensive patients.

\section{Treatment}

Appropriate lifestyle changes are the cornerstone for the prevention and treatment of hypertension. Initiation 
of drug treatment depends on the grade of hypertension and the overall cardiovascular risk assessment. As the main benefits of antihypertensive treatment are due to $\mathrm{BP}$ lowering, the five major drug classes (diuretics, beta blockers, calcium antagonists, angiotensin-converting enzyme inhibitors and angiotensin receptor blockers) are all suitable for initiation and maintenance of antihypertensive treatment, either as monotherapy or in some combinations taking into account the side-effects and specific indications for each drug. The recommended treatment goal is $<140 / 90 \mathrm{mmHg}$.

\section{Specific conditions}

In elderly individuals with SBP $>160 \mathrm{mmHg}$, there is solid evidence to reduce SBP to between 150 and $140 \mathrm{mmHg}$. However, at least in elderly individuals younger than 80 years, antihypertensive treatment may be considered at SBP values $>140 \mathrm{mmHg}$ and aimed at values $<140 \mathrm{mmHg}$, if the individuals are fit and treatment is well tolerated.

Treatment is strongly recommended in diabetic patients when SBP is $>140 \mathrm{mmHg}$, with the aim to lower it consistently to $<140 \mathrm{mmHg}$. DBP target between $80-85 \mathrm{mmHg}$ is supported by the results of two large studies. The simultaneous administration of two RAS blockers (including the renin inhibitor, aliskiren) should be avoided.
In patients with diabetic or non-diabetic renal disease, SBP should be lowered to $<140 \mathrm{mmHg}$ and when overt proteinuria is present values $<130 \mathrm{mmHg}$ may be pursued, provided that changes in eGFR are monitored.

Renovascular artery stenosis secondary to atherosclerosis is relatively frequent, especially in the elderly population, but rarely progresses to hypertension or renal insufficiency. Intervention (renal artery stenting) is at present not recommended in atherosclerotic renal artery stenosis if renal function has remained stable over the past 6-12 months and if hypertension can be controlled by an acceptable medical regimen (class III, level B). There is convincing (though uncontrolled) information favouring this procedure in younger patients with uncontrolled hypertension in fibromuscular hyperplasia.

In case of truly resistant hypertension (confirmed by ambulatory blood pressure measurements) invasive procedures such a renal denervation or baroreceptor stimulation may be considered (class IIb, level C). Until more evidence is available on the long-term efficacy and the safety of this invasive procedures, those techniques can only be performed by experienced operators.

\section{REFERENCE}

1. Mancia G, Fagard R, Narkiewicz K et al. 2013 ESH/ESC guidelines for the management of arterial hypertension: the Task Force for the Management of Arterial Hypertension of the European Society of Hypertension (ESH) and of the European Society of Cardiology (ESC). Eur Heart J 2013; 34: 2159-219. 